\title{
L'aménagement du Rhin de Bâle au lac de Constance
}

\author{
The development of the Rhine \\ between Basle and Lake Constance
}

\author{
PAR L. KOLLY

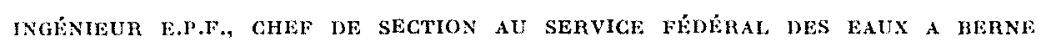

\begin{abstract}
Le plan d'aménagement hydroélectrique du Rhin supérieur entre Bâle el le lac de Constance comporte douze paliers, dont dix sont déja construits. On prévoit d'entreprendre prochainement la réalisation des deux derniers biefs. Enfin, deux anciennes usines devront être reconstruites avec une disposition toute nouvelle. La canalisation du fleuve sur ce secteur permet actuellement une production moyenne annuelle de 3,5 milliards de $\mathrm{kWh}$ qui passera à 4,6 milliards lorsque l'aménagement sera terminé. Environ $45 \%$ de cette énergie sont produits en hiver. L'énergie est répartie entre les Etats riverains dans le rapport des droits de souveraineté sur la force hydraulique, c'est-à-dire à raison de $55 \%$ environ pour la Suisse et de $45 \%$ pour l'Allemagne.

Aujourd'hui, la grande navigation rhénane s'arrête $\dot{a}$ Rheinfelden, environ $20 \mathrm{~km}$ à l'amon de Bàle, après aboir passé par les deux seuls paliers du Rhin supérieur comportant une écluse de natuigation, ceur: de Birsfelden et d'Augst-Wyhlen. Plus à l'amont, les barrages et usines construits dans le Rhin, ou qui le seront prochainement, mettent de longues sections en remous et créent ainsi les conditions nécessaires à la navigation. Pour aménager la voie d'eau, il suffira, pour l'essentiel, d'installer une écluse et des garages au droit de chaque barrage. Cependant, aux donze biefs hydroélectriques que nous benons de mentionner, devront s'ajouter encore denx atres paliers où l'utilisation de la force hydraulique ne pourra être qu'insignifiante, à la chute du Rhin, pour des raisons de protection du site, et a Hemishofen, parce que la chute y est très faible. L'aménagement de la voie nabigable est prévu pour des bateaux mesurant jusqu'a $90 \times 11 \times 2,7 \mathrm{~m}$. On a projeté deux écluses de $165 \mathrm{sur} 12 \mathrm{~m}$ par bief. Le monillage sera d'au moins $3 \mathrm{~m}$ en temps d'étiage et la hauteur libre de $6 \mathrm{~m}$ an-dessus des plus hautes eanx navigables.
\end{abstract}

\begin{abstract}
The hydro-electric development scheme for the lipper Rhine between Basle and lake Constance comprises twelve reaches, ten of which have been constructed already. It is planned to start work on the last two reaches in the near future. Two old power stations are to be rebuilt according to a completely new design.

At the present time, the canalisation of this section of the river results in an average annual outpat of $3,500,000 \mathrm{kWh}$, and this will increase to 4,600,000 $\mathrm{kWh}$ when the scheme is finished. About $45 \%$ of the power is generated in winter and output is shared by the riparian countries in proportion to their sovereign rights concerning water power, i.e., about $55 \%$ for Sixitzerland and $45 \%$ for Germany. At the present day, river traffic on the Rhine ceases at Rheinfelden, about $20 \mathrm{~km}$ upstream of Basle, and uses the Birsfelden and AugstWyhlen locks which are the only two on the tpper Rhine. Long navigable reaches will be created further upstream by existing and planned power stations and dams. The main thing that has to be done to make the river navigable is to construct locks and lock approaches at each dam. Furthermore, two other hydroelectric projects will be added to be twelve that have already been mentioned. They can make use of only an insignificant quantity of the pouner anailable because one of them, the Rhine Falls, is a protected beauty spot and the other, Hemishofen, only provides a very small head. The waterualy is designed to accommodate craft measuring up $1090 \mathrm{~m} \times 11 \mathrm{~m} \times 2.7 \mathrm{~m}$. It is planned to build two $165 \mathrm{~m} \times 12 \mathrm{~m}$ locks on each. The depth of the navigable channel will be at least $3 \mathrm{~m}$ during low water and there will be $6 \mathrm{~m}$ headroom during maximum flood conditions.
\end{abstract}

\section{I. - UTILISATION DE LA FORCE HYDRAULIQUE}

\section{Conditions générales.}

Il est peu d'autres fleuves en Europe qui se prêtent d'une façon aussi favorable à la mise en valeur de l'énergie sur un si long parcours que le Rhin entre Schaffhouse et Bâle.
En effet, le débit du fleuve est important et extraordinairement constant. M. Walser en a analysé, dans un autre article de ce numéro, les caractéristiques hydrologiques. Qu'il nous suffise de rappeler, pour montrer l'influence régulatrice des lacs, que le rapport entre l'étiage 


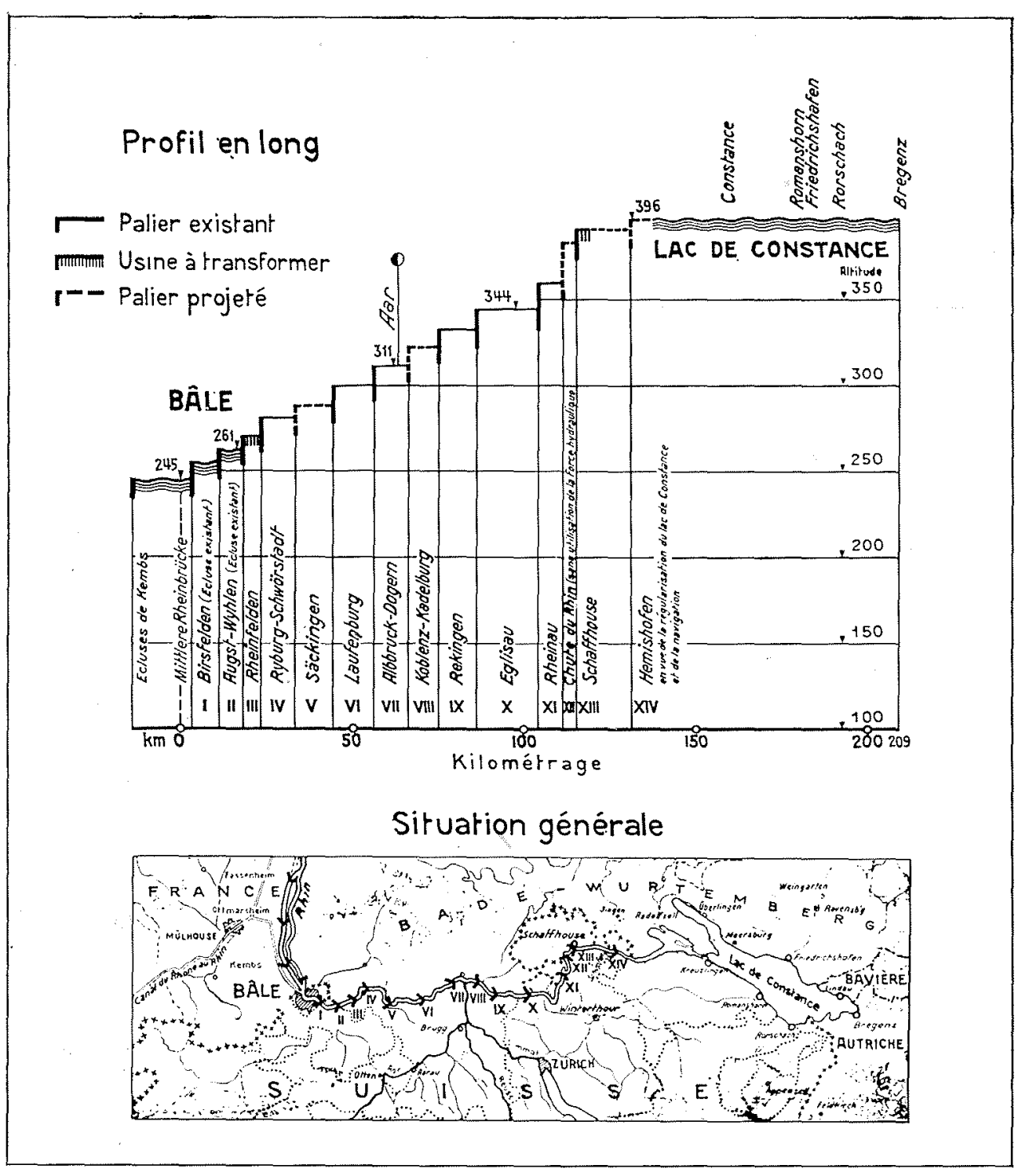

FIG. 1.

Situation générale et profil en long Bâle-lac de Constance le plus faible et la crue la plus forte n'est que de $1 / 10$ à Stein (sortie du lac de Constance) alors qu'il est de $1 / 65$ à l'embouchure dans le lac. L'Aar, qui vient se jeter dans le Rhin près de Koblenz et qui l'alimente d'un volume d'eau qui est, en moyenne, de $25 \%$ supérieur à celui du fleuve à cet endroit, a également régularisé son cours dans d'autres lacs suisses et apporte un débit d'une constance remarquable, si bien qu'elle ne trouble en rien le régime dont nous venons de parler.

En outre, le Rhin, de Stein à Bâle, présente une dénivellation de $150 \mathrm{~m}$ sur une longueur de quelque $140 \mathrm{~km}$, soit une pente moyenne relativement forte d'un peu plus de $1 \%$. Il est vrai que $24 \mathrm{~m}$ de cette différence de niveau sont concentrés à la chute du Rhin et presque totalement soustraits à l'exploitation de la force hydraulique à laquelle on renonce à cet endroit par souci de protection du site admirable, connu bien en dehors de nos frontières. Seule une petite usine existante, qui se cache modestement sur la rive droite, y utilise quelque $25 \mathrm{~m}^{3} / \mathrm{s}$ sans rien enlever à la majesté de la chute et sans dommage pour la beauté du paysage.

Notons également que le charriage d'alluvions, qui pose des problèmes si importants pour l'aménagement d'autres fleuves, est pratiquement inexistant sur ce tronçon et n'a qu'une importance toute locale à l'embouchure de quelques affluents.

Enfin, les conditions topographiques et géologiques, sur lesquelles nous ne pouvons nous ètendre ici, sont en général très propices à la canalisation du fleuve et rares sont les tronçons sur lesquels on se voit obligé d'établir des digues longitudinales de protection à l'amont des barrages. 


\section{Historique.}

Toutes ces conditions extrêmement favorables dont nous venons de parler, ainsi que la situation géographique du Rhin supérieur au centre d'une région de forte consommation, ont provoqué un intérèt tout spécial pour ce tronçon à une époque déjà où le choix de possibilités d'aménagement était encore immense. Ce n'est donc pas par hasard, qu'à la fin du siècle dernier, la première grande usine électrique d'Europe fut construite sur le Rhin supérieur, à Rheinfelden. Cette centrale est encore en service aujourd'hui mais devra ètre bientôt reconstruite entièrement à neuf et disposée différemment (fig. 10). Celle de Schafthouse, qui est plus modeste, mais dale de 1866 déjà, se trouve dans. le même cas.

Avant la première guerre mondiale encore, deux autres grandes usines furent construites à Augst-Wyhlen (fig. 2) ét à Laufenburg. AugstWyhlen présente cette particularité que les parts suisse et allemande à la force hydraulique y sont utilisées séparément dans deux centrales placées symétriquement de part et d'autre du fleuve, le barrage se trouvant au milieu. Cette disposition n'a pas fait école et sur tous les autres paliers l'énergie est exploitée dans une seule centrale.

La réalisation des usines dont nous venons de parler avait eu lieu sans plan d'ensemble bien établi, chaque entreprise utilisant le tronçon du fleuve qui Iui convenait le mieux. Aussi les états riverains reconnurent bientôt qu'il était urgent d'établir un plan général assurant une utilisation rationnelle de tout le Rhin supérieur et tenant compte d'un aménagement ultérieur pour la navigation. Un concours d'idées, ouvert en 1913 par les associations de navigation des 2 pays, se termina en 1920 , après avoir été interrompu par la première guerre mondiale, mais, ce n'est qu'en 1926 que le plan général d'aménagement put être entériné par les deux Etats. Ce plan a subi depuis quelques modifications de détail, il est demeuré valable dans ses grandes lignes.

Au cours des ans, six autres usines hydroélectriques vinrent s'ajouter aux quatre que nous avons déjà mentionnées et, des quatre dernières qui se trouvent encore à l'état de projet, deux sont destinées à remplacer les vieilles installations de Rheinfelden et de Schaffhouse.

En résumé, le projet général d'aménagement hydroélectrique comprend douze usines dont dix sont construites, les usines existantes de Rheinfelden et de Schaffhouse devant être entièrement renovées. Les projets des deux derniers paliers de Säckingen et de Koblenz (fig. 6) sont actuellement tout à fait au point. La pro- cédure de concession pour les quatre dernières usines est en cours et tout laisse prévoir que leur réalisation sera entreprise dans ces prochaines années.

\section{Etat de l'aménagement.}

Le tableau I donne une liste des paliers existants ou projetés et indique, notamment, pour chaque bief, le débit utilisable et la production annuelle.

Ces douze usines, déjà réalisées ou projetées, sont toutes, à l'exception de deux (AlbbruckDogern et Rheinau), des usines-barrages construites dans le fleuve mème. A Albbruck-Dogern on a préféré, quoique cela ne fût pas nécessaire, dériver le débit utilisé dans un canal d'amenée de quelque $4 \mathrm{~km}$; à Rheinau l'usine est placée sur la rive près du barrage et l'eau restituée au fleuve par un tunnel de $400 \mathrm{~m}$ qui coupe une grande boucle du Rhin (fig. 7). A l'état final, à ces douze paliers, s'ajouteront deux biefs, un à l'amont de la chute du Rhin, l'autre près d'Hemishofen, ne comportant qu'un faible remous mais indispensables, le premier à l'aménagement de la voie navigable, le second à la régularisation du lac de Constance et à la navigation.

De l'usine de Rheinfelden, avec ses 20 unités motrices turbinant chacune $30 \mathrm{~m}^{3} / \mathrm{s}$, à l'installation moderne de Birsfelden, équipée de 4 turbines Kaplan de $350 \mathrm{~m} / \mathrm{s}$, les dix usines déjà réalisées illustrent en quelque sorte l'histoire du développement des installations à basse chute dans la première moitié de notre siècle.

La canalisation du fleuve sert donc en premier lieu à l'utilisation de l'énergic. Elle est en même temps, comme nous le verrons plus loin, une condition nécessaịre à l'établissement d'une voie navigable. La production moyenne annuelle lotale des usines existantes se monte à 3,5 milliards de $\mathrm{kWh}$ et elle doit passer à 4,6 milliards lorsque l'aménagement sera terminé. Environ $45 \%$ de cette énergie sont produits en hiver. A l'amont de l'embouchure de l'Aar, le débit utilisé dans les usines modernes est de 500 à $600 \mathrm{~m}^{3} / \mathrm{s}$; à l'aval il est de l'ordre de $1300 \mathrm{~m}^{3} / \mathrm{s}$.

Les installations sont construites et exploitées par des entreprises privées et l'énergie est répartie entre les Etats dans le rapport des droits de souveraineté sur la force hydraulique, c'est-àdire à raison de $55 \%$ environ pour la Suisse et de $45 \%$ pour l'Allemagne.

Les deux pays riverains ont créé en 1919 une commission permanente, chargée de traiter toutes les questions de l'aménagement des usines et les problèmes de navigation qui en résultent. Au vu des avis de cette commission, la 
USINES DU RHIN DE BALE AU LAC DE CONSTANCE

\begin{tabular}{|c|c|c|c|c|c|c|c|}
\hline \multicolumn{2}{|l|}{ USINE } & $\begin{array}{l}\text { Mise } \\
\text { en service } \\
\text { annee }\end{array}$ & $\begin{array}{c}\text { Débit } \\
\text { utilisable } \\
\text { en } \mathrm{m}^{3} / \mathrm{s}\end{array}$ & $\begin{array}{l}\text { Nombre } \\
\text { de groupes }\end{array}$ & $\begin{array}{l}\text { Puissance } \\
\text { installée } \\
\text { en kW }\end{array}$ & $\begin{array}{c}\text { Production } \\
\text { annuelle moync } \\
\text { en } G W h\end{array}$ & $\begin{array}{l}\text { Part suisse } \\
\text { de l'énergie } \\
\text { en } \%\end{array}$ \\
\hline 1. BIRSFELDEN . . . . & étal actuel & 1955 & 1.300 & 4 & 74.800 & $477\left(^{*}\right)$ & 59 \\
\hline 2. AugST-WyHLEN ... & état actuel & 1912 & 800 & 20 & 44.000 & 316 & 50 \\
\hline 3. RHEINFELdeN . . . & etat actuel & 1898 & 614 & 20 & 20.500 & 164 & 50 \\
\hline & projet 1958 & & 1.310 & 6 & 80.400 & $449\left({ }^{*}\right)$ & 50 \\
\hline 4. RYBURG-SCHWÖRSTADT . & état actuel & 1930 & 1.200 & 4 & 108.000 & 732 & 50 \\
\hline 5. Sackingen $\ldots \ldots \ldots$ & projet 1955 & & 1.300 & 4 & 69.000 & $392\left(^{*}\right)$ & 50 \\
\hline 6. Laufenburg . . . . & état actuel & 1914 & 980 & 10 & 77.600 & 554 & 50 \\
\hline 7. AlbBruck-Dogeri & état actuel & 1933 & 1.060 & 3 & 75.000 & 532 & 54 \\
\hline 8. KoBlenz & projet 1957 & & 600 & 3 & 53.200 & $316(*)$ & 50 \\
\hline 9. Rekingen .... & état actuel & 1941 & 560 & 2 & 38.000 & 222 & 50 \\
\hline 10. Eglisau ... & état actuel & 1920 & 400 & 7 & 32.500 & 233 & 93 \\
\hline 11. Rheinau ...... & état actuel & 1956 & 400 & 2 & 40.000 & 215 & 59 \\
\hline 12. Chute du Rhix $(\cdots)$... & ćtat actuel & 1951 & 25 & 1 & 4.400 & 38 & 100 \\
\hline 13. SChaffhouse . . . . & état actuel & 1866,1890 & 135 & 9 & 4.700 & 38 & 100 \\
\hline & projet 1957 & & 425 & 2 & 22.200 & 152 & 93 \\
\hline $\begin{array}{l}\text { Etat actuel. } \\
\text { Utilisable au }\end{array}$ & totai......... & 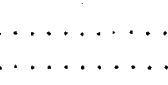 & & & $\begin{array}{l}519.500 \\
719.100\end{array}$ & $\begin{array}{l}3.521 \\
4.628\end{array}$ & \\
\hline
\end{tabular}

(*) Après déduction de la perte provenant de la mise en remous du palier amont.

$\left(^{*}\right)$ Très petite usine, aucun agrandissement projeté (protection des sites).

Suisse et le pays de Bade-Wurtemberg octroient, d'un commun accord, les concessions pour les usines hydrauliques. Ainsi, malgré toutes les difficultés inhérentes à un aménagement de cette envergure dans une région aussi évoluée chevauchant deux Etats, malgré la divergence inévitable des intérêts sur certains points particuliers, malgré deux guerres et la crise économique intermédiaire qui ont fort retardé les travaux, il s'est instauré peu à peu sur le Rhin supérieur une tradition solide de collaboration internationale sincère, compréhensive et amicale que nous nous plaisons à souligner et dont les fruits jouent un rôle important dans l'économie des populations riveraines.

\section{II. - AMENAGEMENT POUR LA NAVIGATION}

\section{Généralités.}

Jusque ver's la fin du siècle dernier, le Rhin entre Bâle et le lac de Constance, malgré plu- sieurs obstacles naturels importants, tels que la chute du Rhin et des rapides, servait surtout de voie navigable. La navigation $\mathrm{y}$ était réglée par des conventions internationales, encore par- 
Fic. 2.

La salle des machines de l'usine d'Augst (1912).

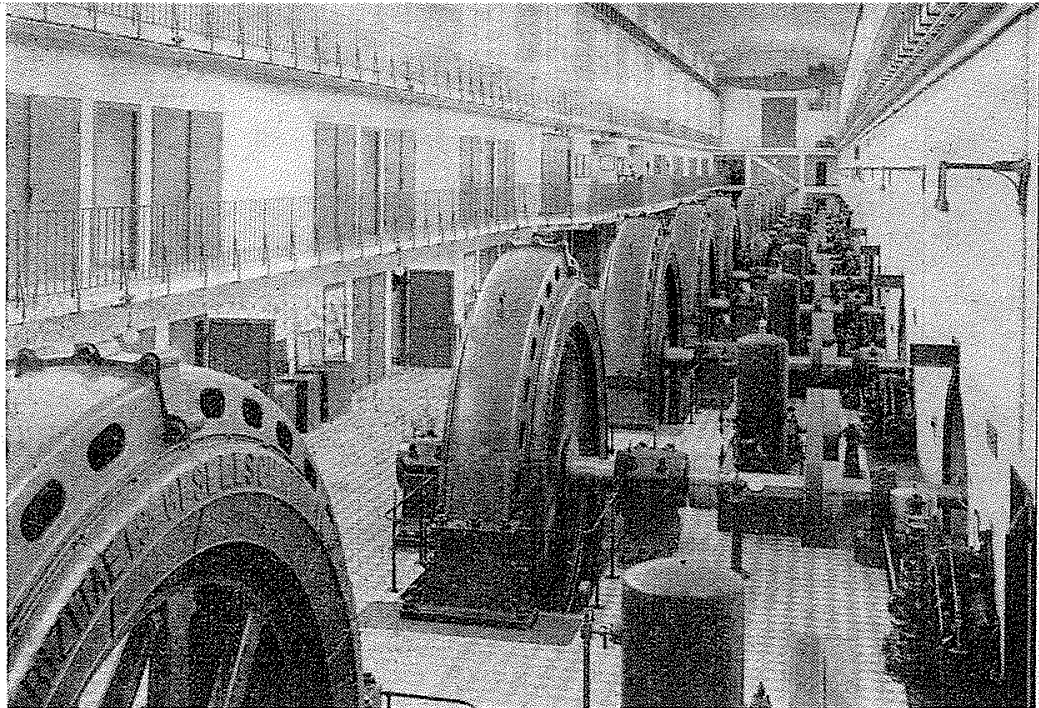

Fig. 3.

Usine de Birsfelden (1955)

A l'amont sur la rive gauche, port de Birsfelden An droit de l'usine les installations de navigation.

Vue aérienne Balair S.A.,

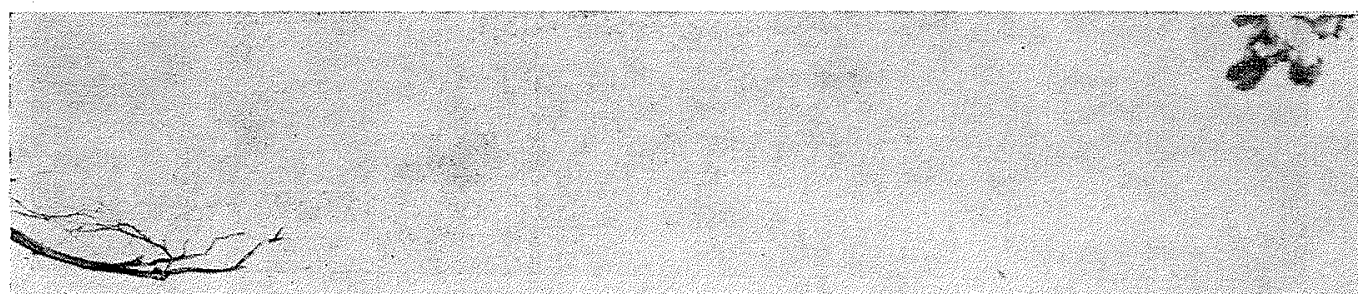

FI(i. 4.

Usine de Birsfelden, vue de l'aval.

(Photo Hinz, Bâle)

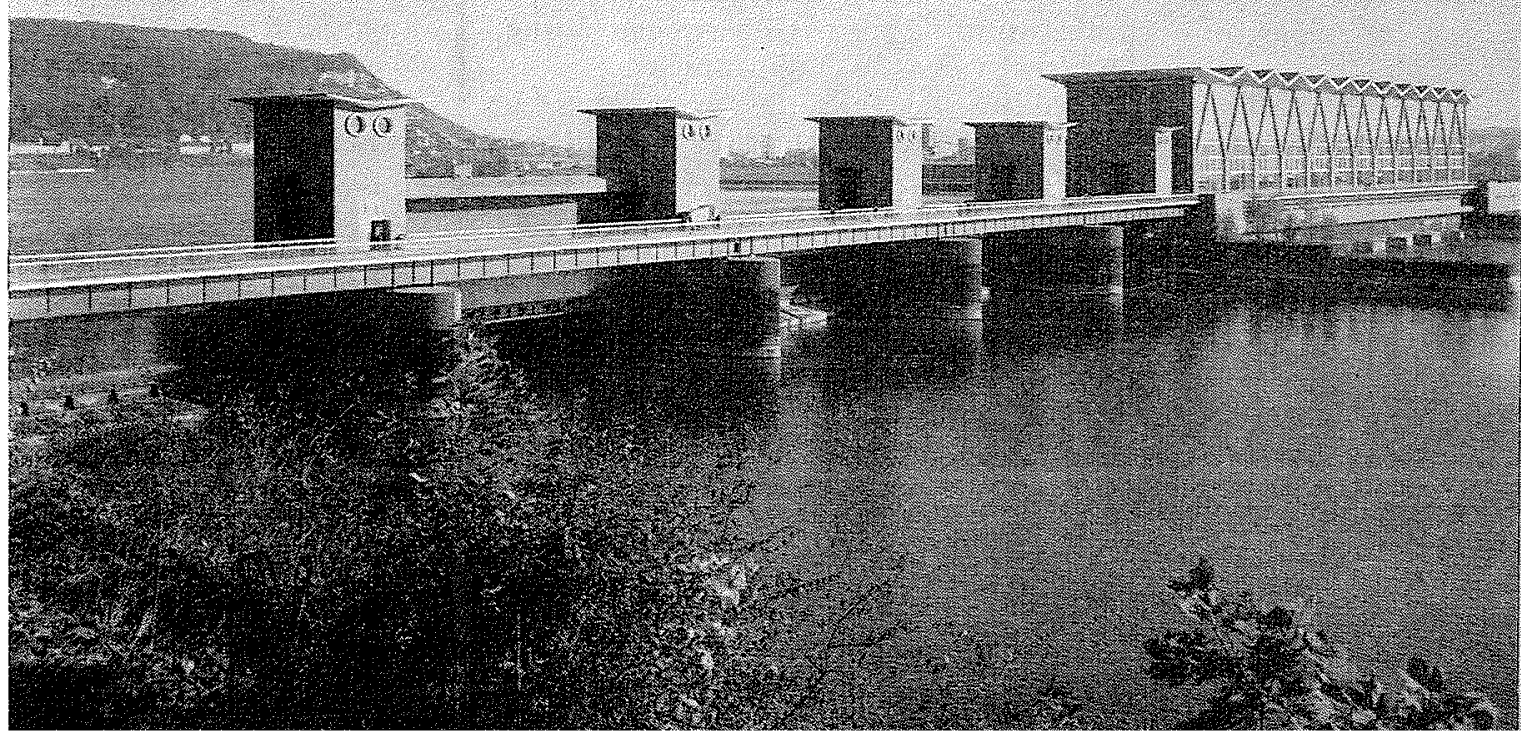




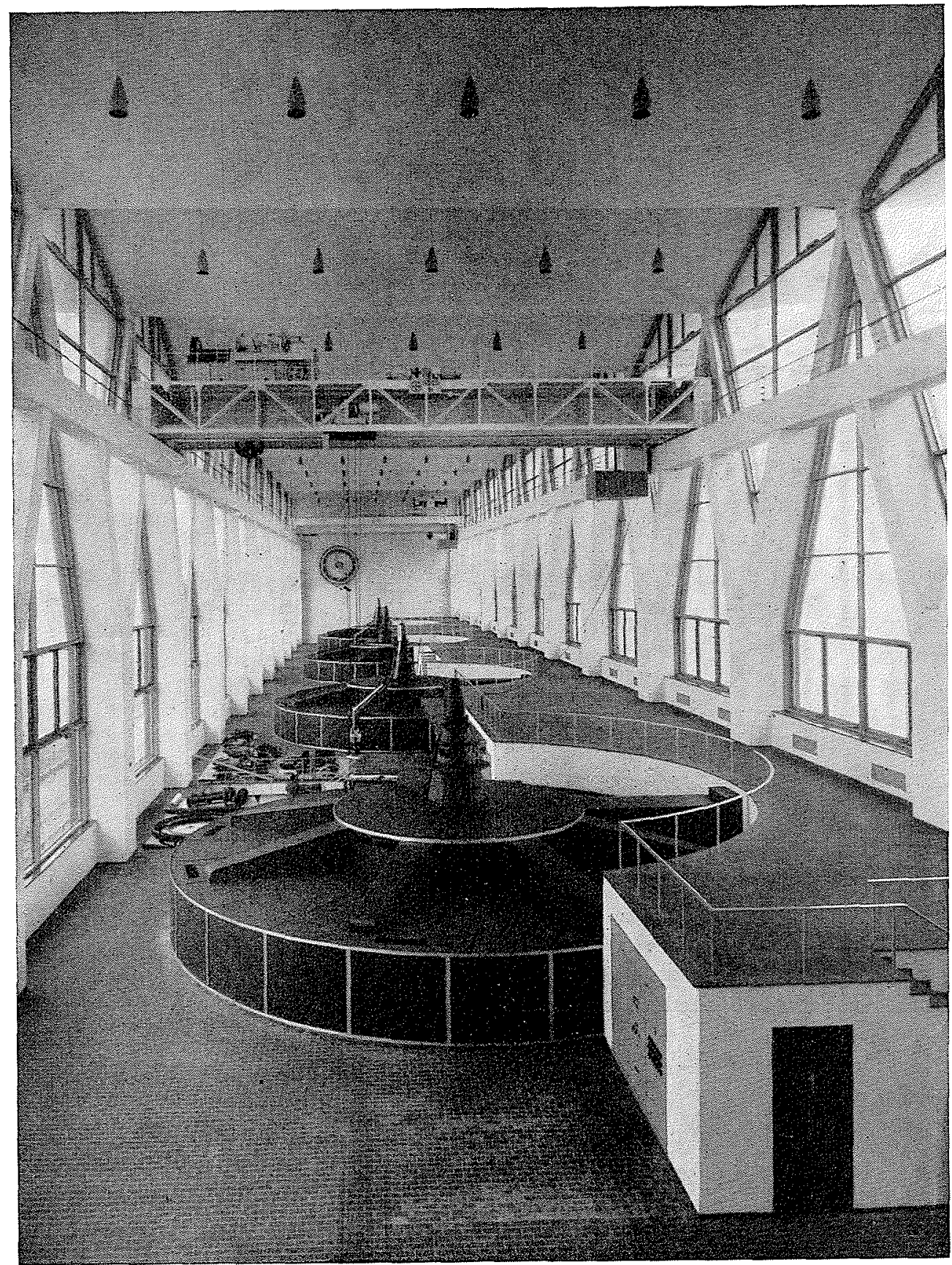

Fig. 5.

Birsfelden : Salle des machines.

(Photo Hinz, Baile)

tiellement en vigueur aujourd'hui. Cependant son importance diminua au fur et à mesure que s'amélioraient les voies de communication terrestres. Aujourd'hui, le trafic des marchandises par bateau est limité à la section BâleRheinfelden.

Au début de ce siècle, en même temps que la navigation perdait de son importance, les progrès de la technique permettaient, comme nous venons de le voir, de construire de grandes usines électriques pour utiliser l'énergie du fleuve. On pouvait dès lors songer à aménager une voie de grande navigation moderne de Bâle jusqu'au lac de Constance. En effet, les barrages construits dans le Rhin, mettent de longues sections en remous et créent ainsi les conditions nécessaires à la grande navigation. Il suffit alors d'installer une écluse et des garages au droit de chaque barrage pour obtenir l'essentiel de la voie navigable. Ici, la question du coût se présente donc de facon plus favorable que pour d'autres cours d'eau qu'on a rendus navigables, tels que le Main et le Neckar, où la navigation doit également supporter une partie des frais 
riverains ont maintenant entrepris cette tâche, ils y travaillent d'un commun accord et comptent la mener" à bien dans le courant de l'année prochaine.

\section{Le projet.}

Comme nous l'avons déjà dit, le projet général comporte l'aménagement de quatorze paliers, à savoir les douze biefs servant à l'utilisation de la force hydraulique et les deux paliers de la chute du Rhin et d'Hemishofen.

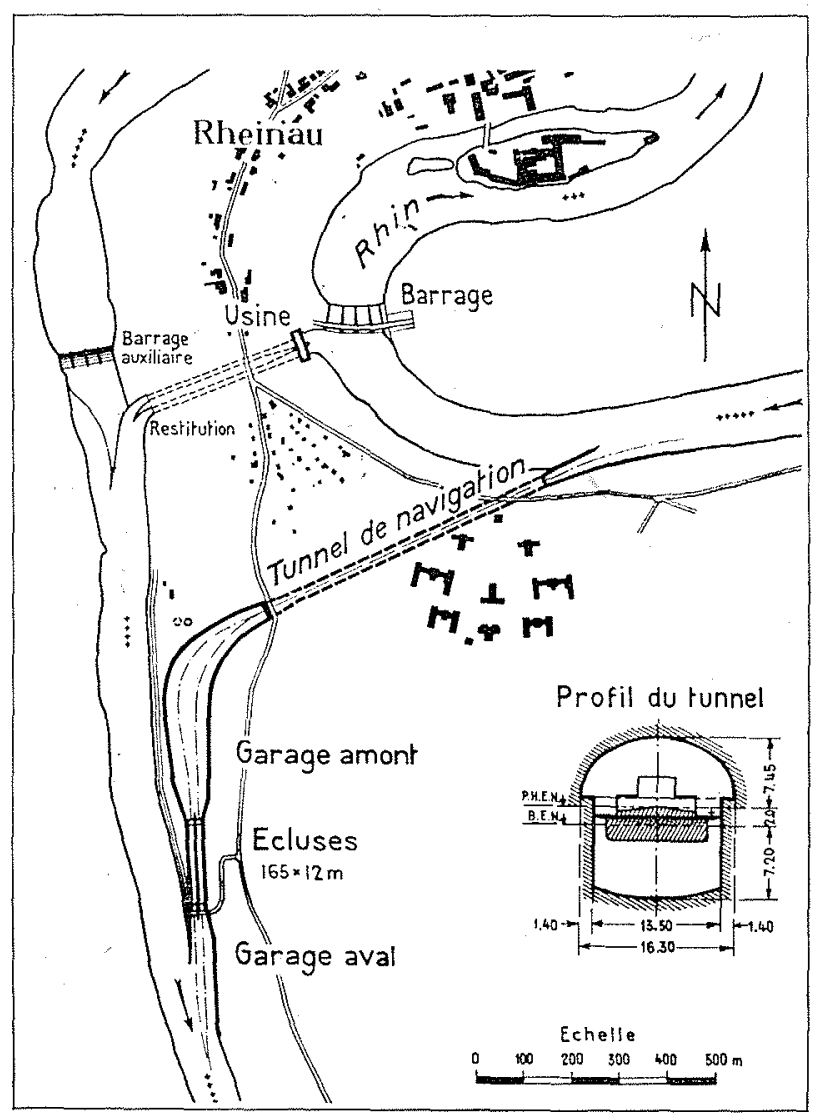

Fig. 8 .

Projet d’aménagement du palier de Rheinau pour la navigation. Situation et profil du tunnel.

Sans vouloir entrer dans le détail des normes qui président à l'établissement du nouveau projet, examinons-en cependant quelques caractéristiques principales.

a) LA FLotTe. - On admet en principe que les bateaux montant actuellement à Bâle conti- nueront leur voyage vers l'amont et qu'on ne construira pas un type spécial pour le Rhin supérieur. L'automoteur sera certainement prédominant sur la nouvelle voie d'eau; les petits convois comprenant une ou deux remorques n'en seront toutefois pas exclus.

Les services techniques des deux pays suivent attentivement les expériences actuellement en cours et tendant a l'introduction du poussage sur le Rhin. Cependant, tout nous fait croire que cette évolution ne viendra en rien bouleverser les normes prévues ci-dessous. En effet, une certaine forme de poussage pourra éventuellement se développer sur le Rhin supérieur, mais ses possibilités seront limitées bien plus par les conditions topographiques et la vitesse du courant que par les dimensions des installations de navigation.

Comme type normal de bateau devant circuler sur la nouvelle voie navigable, on a prévu, conformément aux décisions de la Conférence européenne des ministres des transports, le bâtiment Rhein-Herne $(80 \times 9,5 \times 2,5 \mathrm{~m})$. Cependant, pour bien tenir compte de la composition de la flotte remontant jusqu'à Bâle, on doit également pouvoir naviguer avec des bateaux mesurant jusqu'à $90 \times 11 \times 2,7 \mathrm{~m}$.

b) LES EcLUSEs. - Le projet comporte deux écluses jumelées par palier, mais il reste encore à décider si la deuxième écluse sera construite en même temps que la première.

On a tout d'abord envisagé de donner aux écluses une longueur telle, qu'elles puissent contenir en même temps deux bateaux de $90 \mathrm{~m}$. On aurait done obtenu une longueur utile de $180 \mathrm{~m}$ environ, égale à celle des écluses du Grand Canal d'Alsace et de Birsfelden. Une étude plus poussée a montré que, sur quelques paliers, une telle longueur aurait présenté des désavantages notables quant à la disposition générale des installations et une analyse du trafic nous a amenés à conclure qu'une longueur utile de $165 \mathrm{~m}$ serait la plus rationnelle.

Quant à la largeur, on a maintenu $12 \mathrm{~m}$, dimension déjà prévue dans les projets prócédents et que l'on retrouve sur la plupart des voies navigables reliées au Rhin.

c) Les Garages. - Sur une voie comme le Rhin supérieur, où l'aménagement de presque chaque palier représente un cas d'espèce, il n'est guère recommandable de normaliser la longueur des garages. Dans certains biefs, des garages relativement longs sont faciles à établir et favorables à l'exploitation; dans d'autres ils gênent plutôt l'exercice de la navigation. Ce qui est déterminant, c'est bien plus la nécessité de disposer l'entrée du garage à une distance suffisante du barrage et de telle sorte que les 


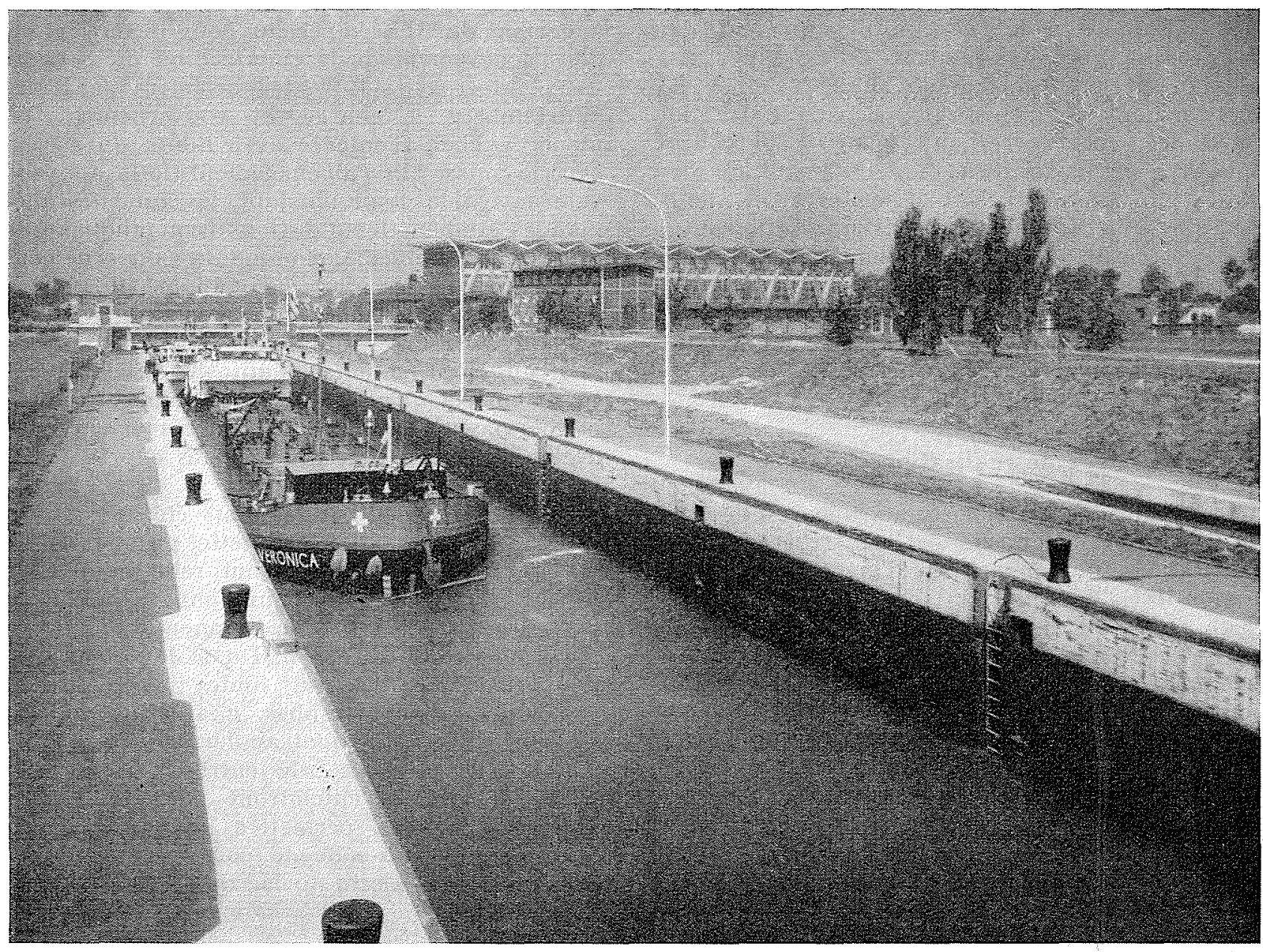

Fici. 9. L Lécluse de Birsfelden.

(Photo Schwilter S.A., Bäle).

bateaux n'aient aucune peine d'entrer ou de sortir des écluses.

En ce qui concerne la largeur, on a prévu jour les garages un minimum de $57 \mathrm{~m}$, e'està-dire trois voies parallèles, suffisantes pour l'aménagement final à deux écluses jumelées.

d) Movillage et tirant d'air. - La profondeur de la voie navigable sera d'au moins $3 \mathrm{~m}$ en lemps d'étiage. Le mouillage minimum sur le busc aval est fixé à $3,50 \mathrm{~m}$ à l'étiage et, sur le busc amont, à $4 \mathrm{~m}$ au-dessous du remous concédé.

Eu égard au grand nombre de ponts existant sur le Rhin et à l'aménagement souvent difficile de leurs accès dans les localités, on a dû se contenter de prévoir un tirant d'air de $6 \mathrm{~m}$ audessus des plus hautes eaux navigables.

\section{Le coût des ouvrages.}

Le coût des travaux, établi sur la base des prix de 1950, s'élevait, en chiffre rond, à 220 millions de francs suisses pour un aménage-

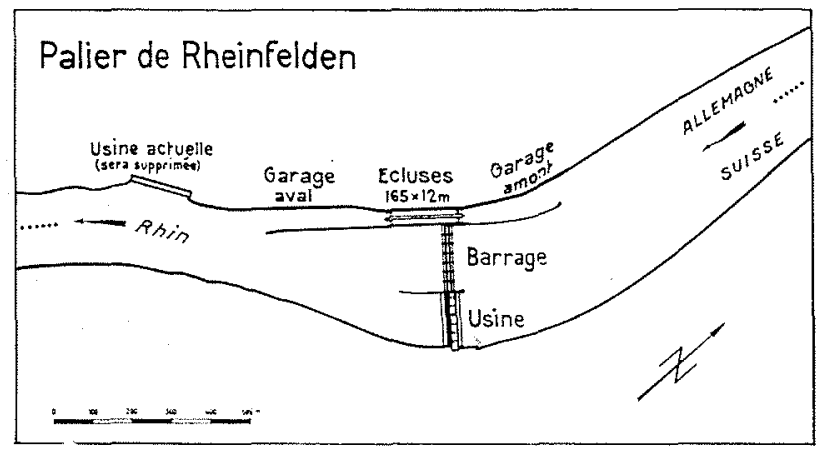

Fig. 10.

Projet d'aménagement c'u palier de Rheinfelden. Situation.

ment comprenant une seule écluse à chaque palier. Ce devis se basait sur le projet publié en 1942. Le nouveau projet remanié comportant des installations plus spacieuses el les prix de construction ayant auggmenté depuis 1950 , il 
faut s'attendre à un certain dépassement de cette somme que nous pouvons estimer approximativement, pour le moment, à $20 \%$ environ. Si l'on tient compte du trajet sur le lac de Constance, cela correspond, pour le parcours Bâle-Bregenz, à un peu plus d'un million de francs par kilomètre, soit à peu près au montant que l'on compte pour construire une route moderne de montagne ou aménager une double voie sur une section des chemins de fer fédéraux. Il est rare de rencontrer des conditions aussi favorables à la construction d'une nouvelle voie navigable.

\section{Perspectives de réalisation.}

A la suite de deux postulats déposés devant les Chambres, le Conseil fédéral a présenté, en mars 1956, un rapport sur l'aménagement de la voie navigable du Rhin supérieur. Dans cette vaste étude fort documentée, le gouvernement suisse s'attache tout spécialement à élucider les conséquences économiques qu'entraînerait, pour notre pays, l'exécution de l'entreprise. La discussion du rapport devant les deux Chambres a eu lieu dans une ambiance que l'on peut certes qualifier de favorable à la réalisation du projet.

Du côté allemand, soit le ministre fédéral des transports, soit le gouvernement du Pays de Bade-Wurtemberg ont, à plusieur's reprises, exprimé tout l'intérêt qu'ils portent à un amé- nagement du Rhin supérieur pour la navigation dès l'achèvement de la canalisation du Neckar. Au printemps de 1955 , le parlement de BadeWurtemberg a chargé le gouvernement de mener à chef, d'entente avec Bonn, un accord avec la Suisse sur l'aménagement de la nouvelle voie navigable. Pour l'instant, les autorités allemandes étudient les questions économiques que soulève le problème, comme le gouvernement suisse l'a déjà fait dans son rapport de 1956 .

L'Autriche également, qui aurait un avantage évident à se créer ainsi un débouché vers la mer du Nord, a déjà manifesté officiellement le vif intérêt qu'elle porte à l'aménagement de la voie navigable du Rhin supérieur et elle s'est déclarée prête à soutenir tous les efforts entrepris pour sa réalisation.

Le climat général n'a jamais été aussi favorable à la réalisation de cette grande cuvre qui sera la prolongation naturelle de l'artère fluviale rhénane dont chacun connaît toute l'importance dans le cadre de l'économie des transports de l'ouest européen. On a donc tout lieu de penser que, dès que toutes les conditions préalables seront remplies, c'est-à-dire lorsque la construction des quatres derniers paliers du Rhin supérieur aura été entreprise, les trois Etats intéressés s'entendront pour réaliser la voie navigable et permettre ainsi à la flotte rhénane internationale de pousser son activité jusqu'au vaste bassin du lac de Constance, pour le plus grand bien de l'économie des populations liveraines.

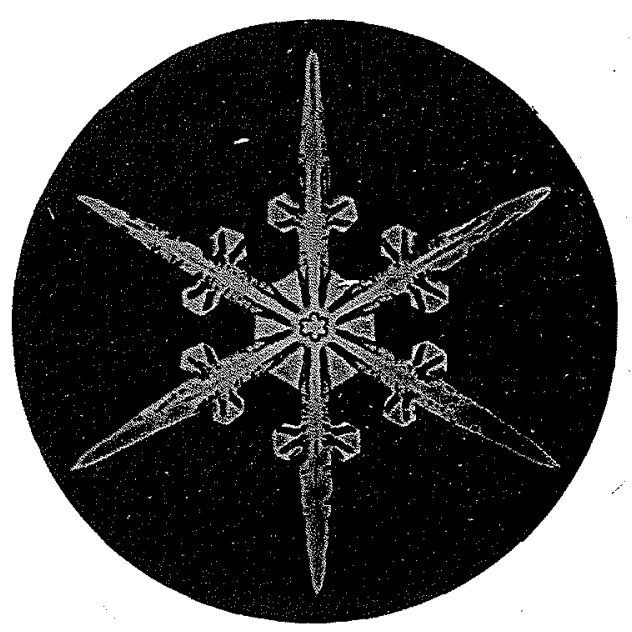

